\title{
Effects of glucose-dependent insulinotropic polypeptide receptor knockout and a high-fat diet on cognitive function and hippocampal gene expression in mice
}

\author{
RACHAEL R. LENNOX, CHARLOTTE MOFFETT, DAVID W. PORTER, \\ NIGEL IRWIN, VICTOR A. GAULT and PETER R. FLATT \\ School of Biomedical Sciences, The SAAD Centre for Pharmacy and Diabetes, University of Ulster, \\ Coleraine, Londonderry BT52 1SA, Northern Ireland, UK
}

Received June 2, 2014; Accepted February 19, 2015

DOI: $10.3892 / \mathrm{mmr} .2015 .3447$

\begin{abstract}
It has been previously demonstrated that compromise of glucose-dependent insulinotropic polypeptide receptor (GIPR) action and chronic consumption of a high-fat diet can independently impair memory and learning ability, however, the underlying pathology remain to be elucidated. The present study investigated the effects of GIPR knockout (KO), alone and in combination with a high-fat diet, on aspects of cognitive function and hippocampal gene expression in mice. In object recognition tests, normal mice exhibited effective memory, preferring to investigate the novel over the familiar object. However, wild-type (WT) mice fed a high-fat diet and GIPR $\mathrm{KO}$ mice fed a standard or high-fat diet demonstrated no such discrimination, suggesting the impairment of memory function. This decline in cognitive function was associated with marked changes in the expression levels of hippocampal genes involved in memory and learning. The chronic consumption of a high-fat diet decreased the hippocampal gene expression levels of mammalian target of rapamycin (mTOR), neurotrophic tyrosine kinase receptor type 2 (NTRK2) and synaptophysin. Notably, the GIPR KO mice fed a high-fat diet exhibited no reduction in the hippocampal expression of synaptophysin expression, however, the GIPR KO mice fed a standard rodent maintenance diet exhibited reduced hippocampal expression of mTOR compared with the WT controls. These data highlighted the importance of intact GIPR signalling and dietary composition in modulating memory and learning, and hippocampal pathways involved in the maintenance of synaptic plasticity, including mTOR and NTRK2, appear to be key in this regard.
\end{abstract}

Correspondence to: Dr Nigel Irwin, School of Biomedical Sciences, The SAAD Centre for Pharmacy and Diabetes, University of Ulster, Cromore Road, Coleraine, Londonderry BT52 1SA, Northern Ireland, UK

E-mail: n.irwin@ulster.ac.uk

Key words: cognitive function, glucose-dependent insulinotropic polypeptide, high-fat diet, hippocampus, gene expression

\section{Introduction}

Glucose-dependent insulinotropic polypeptide (GIP) is an incretin hormone, secreted by enteroendocrine K-cells, with established effects on insulin secretion and pancreatic $\beta$-cell function (1). Furthermore, GIP and its receptor (GIPR) have been identified in several areas of the brain, including the cortex, cerebellum and hippocampus (2-4). However, the role of GIP within the central nervous system remains to be elucidated. To date, GIP has been observed to exhibit a number of neuroprotective effects, inducing progenitor cell proliferation, improving learning and memory and enhancing synaptic plasticity $(4,5)$. Indeed, the genetically-induced overexpression of GIP in mice improves their cognitive function (6). In addition, mice with a targeted deletion of the GIPR have been reported to have less than half number of newly proliferating cells in the hippocampus (4) and exhibit impaired learning and memory, compared with the wild-type (WT) controls (7). Overall, these preliminary results suggested that the GIPR is important in cognitive function in the central nervous system, however, the underlying mechanisms remain to be elucidated.

Evidence from previous studies has highlighted the association between obesity, type 2 diabetes and cognitive dysfunction (8-10). Previous studies in genetically obese ob/ob mice, Zucker fa/fa rats and high-fat diet fed obese-diabetic mice, have consistently reported impaired performance in learning and memory tests (11-14). Furthermore, a clear association has been demonstrated between chronic high-fat feeding, the increased secretion of GIP and the development of insulin resistance and obesity-associated diabetes (15). With the incidence of human obesity-associated diabetes expected to rise significantly, the occurrence of cognitive deficit disorders, including Alzheimer's disease, is also likely to increase (16). Therefore, it is necessary to elucidate the underlying pathological mechanisms and putative associations between a high-fat diet, GIPR signalling and cognitive decline.

The present study evaluated the role of GIPR signalling and a chronic high-fat diet, alone and in combination, on several aspects of cognitive function in mice. This included investigating learning and memory through object recognition tests (ORTs) and assessing the mechanisms involved by 
analysing the expression levels of key hippocampal genes, which are involved in learning and memory formation. These investigations aimed to determine the importance of GIPR signalling and dietary composition in controlling the fundamental aspects of memory and learning.

\section{Materials and methods}

Animals. Male C57BL/6 mice with genetic deletion of the GIPR were used in the present study, in addition to WT controls. These mice (32 mice in total, 10 GIPR KO and 10 WT mice on a high fat diet, 6 GIPR KO and WT mice on a normal diet; 6-8 weeks old; $n=6-10$ ) were derived from an in-house breeding colony, as described previously $(17,18)$ and were age-matched and housed in an air-conditioned room at $22 \pm 2^{\circ} \mathrm{C}$ with a $12 \mathrm{~h}$ light/dark cycle (08:00-20:00 h). The experimental animals had free access to drinking water, a standard rodent diet (10\% fat, $30 \%$ protein and $60 \%$ carbohydrate; percentage of total energy of $12.99 \mathrm{kj} / \mathrm{g}$; Trouw Nutrition, Cheshire, UK) or a high-fat diet (45\% fat, $20 \%$ protein and 35\% carbohydrate; percent of total energy of $26.15 \mathrm{kj} / \mathrm{g}$; Special Diets Service, Essex, UK), as appropriate. Prior to commencement of experiments, all the mice were weighed and maintained on their respective diets for 120 days. The WT animals, which were fed a high-fat diet exhibited significantly increased body weight compared with the controls. All experiments were performed according to UK Home Office Regulations (UK Animals Scientific Procedures Act 1986), the 'Principles of Laboratory Animal Care' (NIH publication No. 86-23, revised 1985) and were approved by the University of Ulster Animal Ethics Review Committee.

Object recognition. At the end of the dietary intervention, the groups of mice were subjected to ORTs, as described previously (14). Briefly, the mice were initially habituated to the exploratory arena (58 $\mathrm{cm}$ diameter, $38 \mathrm{~cm}$ high) for 5 mins. Two identical random objects ( 2 marbles, $2.5 \mathrm{~cm}$ diameter or 2 die, $1.2 \mathrm{~cm}$ side length) were subsequently placed in the centre of the arena and, following $4 \mathrm{~h}$ exposure (the acquisition phase), one of the two objects was replaced by a novel object (a marble or dice) and the duration spent exploring each object during a 5 min trial phase was determined using a computerised tracking system (Tracker, Biosignal Group, New York, USA). The recognition index (RI), which was designated as the period of time spend exploring the novel object as opposed to the familiar object, was calculated, as described previously (19).

Hippocampal gene expression. Animals were sacrificed by lethal inhalation of $\mathrm{CO}_{2}$ followed by cervical dislocation. Hippocampal tissue was excised at the end of the dietary intervention period by careful surgical excision, snap frozen and processed for gene expression by reverse transcription-quantitative polymerase chain reaction (RT-qPCR) following total RNA extraction. The total RNA was extracted and purified using Tripure Isolation reagent (Roche Diagnostics, West Sussex, UK). The concentration and purity of the extracted RNA was determined using a nanophotometer (NanoPhotometer ${ }^{\mathrm{TM}}$ Pearl; Implen, Munich, Germany) at an absorbance of $260 \mathrm{~nm}$. The cDNA was synthesised (final concentration, $1.5 \mu \mathrm{g})$ using a Transcriptor First Strand cDNA Synthesis kit (Roche Diagnostics) according to the manufacturer's instructions. Gene expression analysis was performed using a Roche RealTime ready qPCR assay, LightCycler 480 Probes Master and a hot start reaction mix (Roche Diagnostics). The following RT-qPCR target specific primers were used: Glucagon-like peptide-1 receptor (GLP-1R); mammalian target of rapamycin (mTOR); neurotrophic tyrosine kinase receptor type 2 (NTRK2); sirtuin 1 (SIRT1); synaptophysin (SYP) and vascular endothelial growth factor (VEGF). The gene expression was normalised to the expression of hypoxanthine guanine phosphoribosyl transferase. Briefly, the specific primer (10 pmol in $20 \mu \mathrm{l}$ reaction volume, $0.5 \mathrm{pmol} / \mu \mathrm{l})$ and cDNA (40 ng/ $\mu \mathrm{l}$.) were added to each well (containing fluorescein-labelled short hydrolysis probes and PCR-grade water) to a final reaction volume of $10 \mu 1$. The PCR conditions were $95^{\circ} \mathrm{C}$ for $10 \mathrm{~min}$, followed by cDNA amplification for 45 cycles with $95^{\circ} \mathrm{C}$ denaturation for $10 \mathrm{sec}, 60^{\circ} \mathrm{C}$ annealing for $30 \mathrm{sec}$ and $72^{\circ} \mathrm{C}$ elongation for $1 \mathrm{sec}$, followed by $30 \mathrm{sec}$ cooling at $40^{\circ} \mathrm{C}$. The relative quantification was calculated using the $2^{-\Delta \Delta C T}$ method, to determine the differences in gene expression levels between the samples (20).

Statistical analysis. One-way analysis of variance, followed by Newman-Keuls post-hoc test were used for statistical analysis using Prism 5 software (Graph-Pad Prism ${ }^{\circledR}$, San Diego, CA, USA). The data are expressed as the mean \pm standard error of the mean. $\mathrm{P}<0.05$ was considered to indicate a statistically significant difference.

\section{Results}

Effects of GIPR KO and high-fat diet alone, and in combination, on object recognition. In the ORT, no difference or bias was observed in the RI between any of the groups of mice during the acquisition period. During the trial phase, the WT mice fed a normal diet had significantly $(\mathrm{P}<0.05)$ increased RI compared with the acquisition phase (Fig. 1A), indicating a preference to examine the novel object. By contrast, the WT mice fed a high-fat diet and the GIPR KO mice fed either the standard maintenance or the high-fat diet failed to discriminate between the novel and the familiar object during the trial phase (Fig. 1B-D).

Effects of GIPR KO and high-fat feeding alone, and in combination, on hippocampal gene expression levels. The assessment of the hippocampal gene expression levels revealed that a high-fat diet significantly decreased the expression levels of mTOR, NTRK 2 and SYP $(\mathrm{P}<0.001, \mathrm{P}<0.05$ and $\mathrm{P}<0.05$; respectively) in the WT mice (Fig. 2A-C). Notably, the GIPR KO mice fed a standard rodent diet also exhibited significantly $(\mathrm{P}<0.05)$ decreased hippocampal expression of mTOR compared with the WT mice (Fig. 2A). Similarly, the GIPR KO mice fed a high-fat diet exhibited significantly $(\mathrm{P}<0.05)$ decreased expression of mTOR, however, this was higher $(\mathrm{P}<0.001)$ compared with the WT mice fed a high-fat diet (Fig. 2A). In addition, the GIPR KO mice fed a high-fat diet also exhibited decreased hippocampal gene expression of NTRK2 compared with the WT controls (Fig. 2B). The expression levels of hippocampal GLP-1R, SIRT1 and VEGF 
A

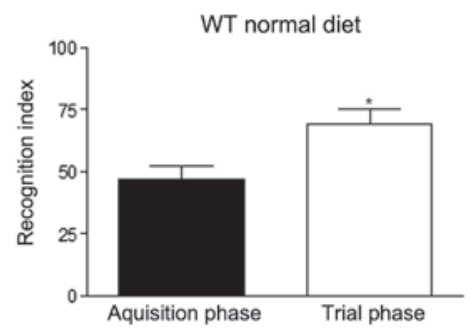

C

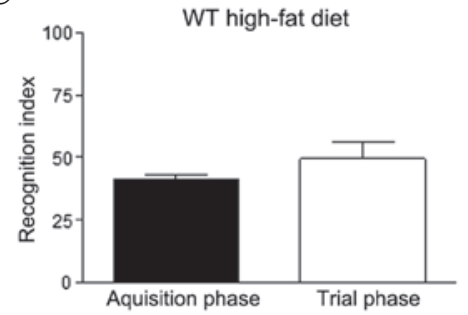

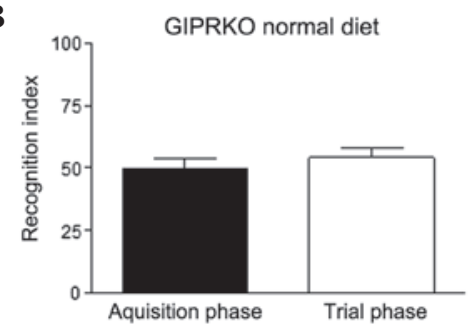

D

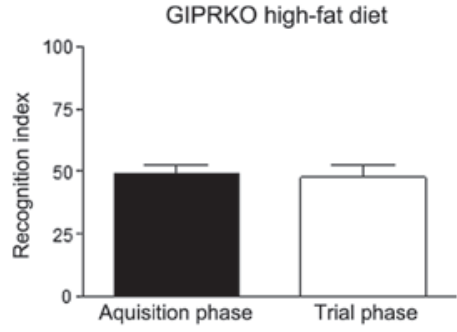

Figure 1. Effects of (A) normal diet, (B) GIPR KO, (C) high-fat feeding and (D) GIPR KO in combination with a high-fat feeding on recognition memory. Experiments were performed following 120 days of dietary intervention. An object recognition test (5 min) was performed using two familiar objects during an acquisition phase and following introduction of a novel object $4 \mathrm{~h}$ later for the trial phase. The recognition index was the percentage of time spent exploring the novel, vs. familiar object. Data are expressed as the mean \pm standard error of the mean for 10 mice ( $\mathrm{P}<0.05$, compared with the acquisition phase). WT, wild-type; GIPR, glucose-dependent insulinotropic polypeptide receptor; KO, knockout.
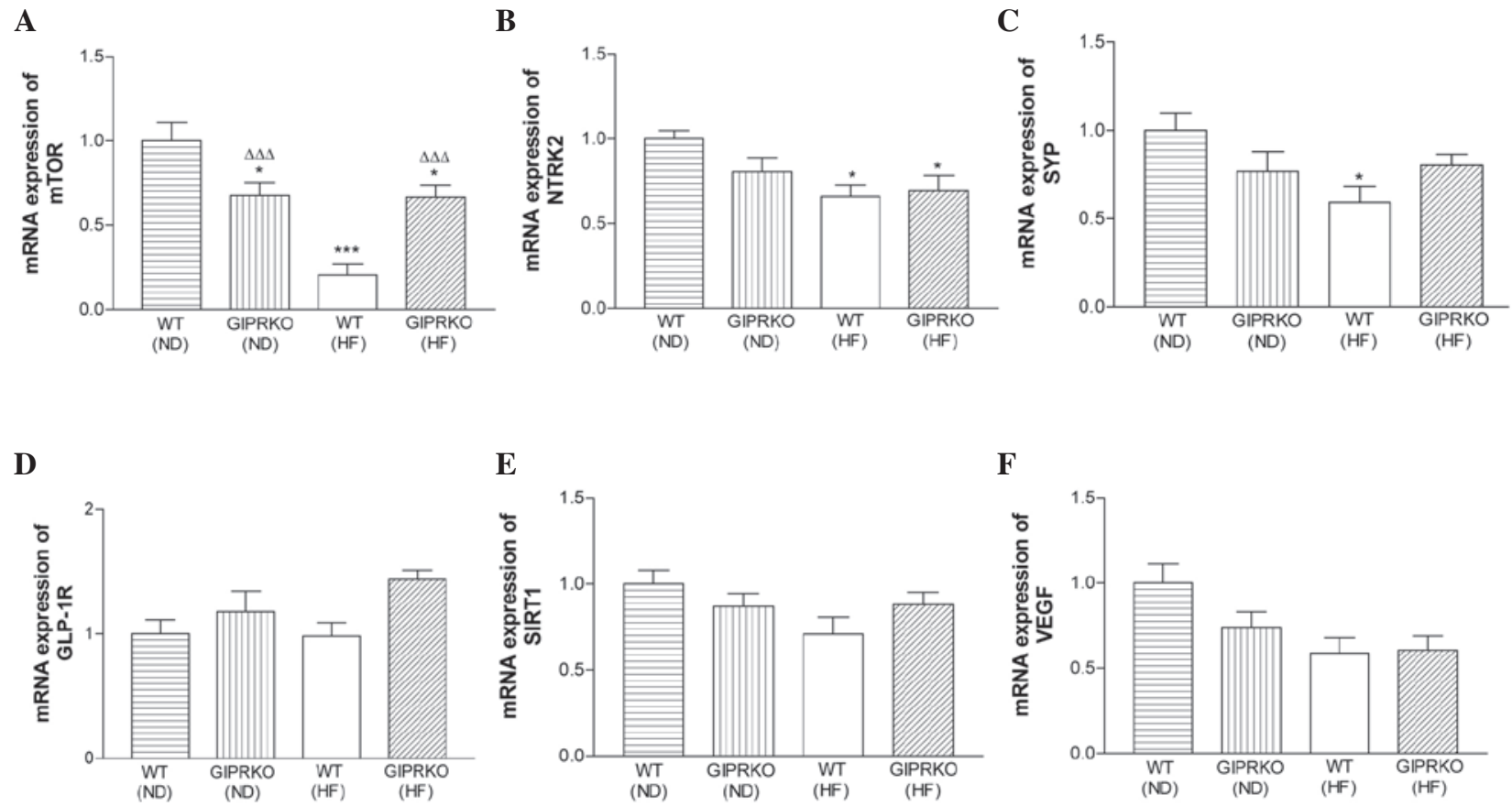

Figure 2. Effects of GIPR KO alone or in combination with a high-fat diet on hippocampal gene expression. The mRNA expression levels of (A) mTOR, (B) NTRK2, (C) SYP, (D) GLP-1R, (E) SITR1 and (F) VEGF were examined following 120 days of dietary intervention. The data are expressed as the mean \pm standard error of the mean for six mice $\left({ }^{*} \mathrm{P}<0.05\right.$ and ${ }^{* * *} \mathrm{P}<0.001$, compared with WT mice fed a normal diet; ${ }^{\Delta \Delta \Delta} \mathrm{P}<0.001$, compared with WT mice fed a high-fat diet). WT, wild-type; GIPR, glucose-dependent insulinotropic polypeptide receptor; KO, knockout; ND, normal diet; HD, high-fat diet; mTOR, mammalian target of rapamycin; NTRK2, neurotrophic tyrosine kinase receptor type 2; SYP, synaptophysin; GLP-1R, glucagon-like peptide 1 receptor; SITR1, sirtuin 1; VEGF, vascular endothelial growth factor.

were not significantly different in any of the groups of mice (Fig. 2D and E).

\section{Discussion}

Chemical and genetic reduction of GIPR signalling, or chronic consumption of a high-fat diet, have been demonstrated independently to impair learning and memory ability $(4,7,12,13)$. However, the precise mechanisms and pathologies underlying these phenomena remain to be elucidated. The present study investigated the effects of genetic GIPR KO alone, and in combination with a chronic high-fat diet, on recognition memory and the expression levels of a panel of hippocampal genes, which are involved in maintaining normal cognitive function. 
The ORT is a popular and informative tool used for assessing recognition memory in rodents (21). In the present study, WT mice maintained on a standard rodent maintenance diet exhibited normal recognition memory, preferring to investigate the novel object during ORTs. In accordance with previous findings, the GIPR KO mice or mice fed a chronic high-fat diet demonstrated significantly impaired recognition memory $(7,13)$. Therefore, it was not unexpected that the GIPR KO mice fed a high-fat diet also exhibited markedly reduced memory function. However, the absence of additive detrimental effects of GIPR annulment combined with a high-fat diet on recognition memory, suggested that similar pathways may be involved in mediating this action, although this requires further investigation.

Normal hippocampal function is particularly important for the preservation of recognition memory (22). Experimental evidence suggested that the deleterious effect of chronic high-fat feeding on learning and memory is associated with altered hippocampal gene expression levels (23). Furthermore, the GIPR KO mice exhibited significant reductions in progenitor cell proliferation in the dentate gyrus of the hippocampus (7). Notably, GIPR KO mice and mice fed a chronic high-fat diet have previously been demonstrated to inhibit long term potentiation (LTP) in the hippocampus, the major cellular mechanism underlying learning and memory $(7,13)$. Therefore, to extend this previous investigation, the present study investigated the effects of GIPR KO alone, and in combination with a high-fat diet, on the expression levels of hippocampal genes, which are known to be involved in cognitive function.

In the present study, the hippocampal expression of mTOR, a protein kinase with an established role in the maintenance of LTP (24), was significantly reduced by GIPR KO and a high-fat diet. Therefore, the expression of mTOR and subsequent activity clearly have major implications on the cognitive ability of each of these groups of mice. Notably, GIPR KO partially reversed the severe detrimental effect of a high-fat diet on the expression of mTOR, although the expression levels remained reduced compared with the WT controls. This may appear marginally contradictory, however, it may be linked to the opportunity for lifelong adaptive mechanisms in genetic KO animals (17). GLP-1 action, also associated with positive effects on cognition (13), has previously been observed to be upregulated in GIPR KO mice (17). In the present study, increased hippocampal expression of GLP-1R was observed in each group of GIPR KO mice, however was not statistically significant. Similarly, the hippocampal expression of SYP was significantly reduced by a chronic high-fat diet, however, concurrent GIPR KO restored the expression of SYP almost to normal levels. SIRT1 has previously been revealed as an important factor for normal cognitive function and object recognition $(23,25)$, however, the hippocampal expression of SIRT1 was unaffected by GIPR KO or a high-fat diet in the present study.

NTRK2, a gene encoding for the tyrosine kinase receptor, TrkB, on neuronal cells, is important in hippocampal synaptic plasticity and neurogenesis (26). The present study revealed that the chronic consumption of a high-fat diet by the WT or GIPR KO mice significantly reduced the hippocampal expression of NTRK2. Therefore, a potential mechanism for the reported impairment of neurogenesis by high-fat feeding (27) may be associated with a reduced expression of NTRK2, which may ultimately decrease the activity of its ligand, brain-derived neurotrophic factor (BDNF). BDNF has well recognised beneficial actions on the growth and differentiation of neurons and synapses (28). Notably, the expression of VEGF, which is important in hippocampal neurogenesis $(29,30)$, was not significantly reduced in all GIPR KO and high-fat fed mice. Taken together, GIPR KO deletion and a chronic high-fat diet may impair hippocampal neurogenesis via complementary pathways.

In conclusion, GIPR KO and chronic consumption of a high-fat diet exhibited negative effects on hippocampal-dependent recognition memory. The pathways involved in the maintenance of hippocampal synaptic plasticity, including SYP, NTRK2 and particularly mTOR, appeared to be key in this respect. In addition, the results suggested that a deficit in hippocampal neurogenesis may also be important in the observed reduction in recognition memory. These findings highlight the importance of GIPR signalling and dietary content for the maintenance of normal cognitive function.

\section{Acknowledgements}

The presentstudy was supported by the Department of Education and Learning, Northern Ireland, and a grant from the European Foundation for the Study of Diabetes/GlaxoSmithKline. The GIPR KO mice were derived from breeding pairs, which were kindly supplied by Professor B Thorens (Lausanne, Switzerland).

\section{References}

1. Gault VA, O'Harte FP and Flatt PR: Glucose-dependent insulinotropic polypeptide (GIP): anti-diabetic and anti-obesity potential? Neuropeptides 37: 253-263, 2003.

2. Usdin TB, Mezey E, Button DC, Brownstein MJ and Bonner TI: Gastric inhibitory polypeptide receptor, a member of the secretin-vasoactive intestinal peptide receptor family, is widely distributed in peripheral organs and the brain. Endocrinology 133: 2861-2870, 1993

3. Nyberg J, Anderson MF, Meister B, et al: Glucose-dependent insulinotropic polypeptide is expressed in adult hippocampus and induces progenitor cell proliferation. J Neurosci 25: 1816-1825, 2005.

4. Nyberg J, Jacobsson C, Anderson MF and Eriksson PS: Immunohistochemical distribution of glucose-dependent insulinotropic polypeptide in the adult rat brain. J Neurosci Res 85: 2099-2119, 2007.

5. Faivre E, Hamilton A and Hölscher C: Effects of acute and chronic administration of GIP analogues on cognition, synaptic plasticity and neurogenesis in mice. Eur J Pharmacol 674: 294-306, 2012.

6. Ding KH, Zhong Q, Xie D, et al: Effects of glucose-dependent insulinotropic peptide on behavior. Peptides 27: 2750-2755, 2006.

7. FaivreE, Gault VA, Thorens B and HölscherC: Glucose-dependent insulinotropic polypeptide receptor knockout mice are impaired in learning, synaptic plasticity and neurogenesis. J Neurophysiol 105: 1574-1580, 2011.

8. Cukierman T, Gerstein HC and Williamson JD: Cognitive decline and dementia in diabetes-systematic overview of prospective observational studies. Diabetologia 48: 2460-2469, 2005.

9. Luchsinger J, Reitz C, Patel B, et al: Relation of diabetes to mild cognitive impairment. Arch Neurol 64: 570-575, 2007.

10. Strachan MWJ, Reynolds RM, Frier BM, Mitchell RJ and Price JF: The relationship between type 2 diabetes and dementia. Br Med Bull 88: 131-146, 2008.

11. Greenwood CE and Winocur G: Glucose treatment reduces memory deficits in young adult rats fed high-fat diets. Neurobiol Learn Mem 75: 179-189, 2001. 
12. Biessels GJ and Gispen WH: The impact of diabetes on cognition: what can be learned from rodent models? Neurobiol Aging 26 (Suppl 1): 36-41, 2005.

13. Gault VA, Porter WD, Flatt PR and Hölscher C: Actions of exendin-4 therapy on cognitive function and hippocampa synaptic plasticity in mice fed a high-fat diet. Int J Obes 34: 1341-1344, 2010.

14. Porter WD, Flatt PR, Hölscher C and Gault VA: Liraglutide improves hippocampal synaptic plasticity associated with increased expression of Mash1 in ob/ob mice. Int J Obes 37 : 678-684, 2013.

15. Irwin $\mathrm{N}$ and Flatt PR: Evidence for beneficial effects of compromised gastric inhibitory polypeptide action in obesity-related diabetes and possible therapeutic implications. Diabetologia 52: 1724-1731, 2009

16. Tang J, Pei Y and Zhou G: When aging-onset diabetes is coming across with Alzheimer disease: comparable pathogenesis and therapy. Exp Gerontol 48: 744-750, 2013.

17. Preitner F, Ibberson M, Franklin I, et al: Thorens, Gluco-incretins control insulin secretion at multiple levels as revealed in mice lacking GLP-1 and GIP receptors. J Clin Invest 113: 635-645, 2004

18. Mieczkowska A, Irwin N, Flatt PR, Chappard D and Mabilleau G: Glucose-dependent insulinotropic polypeptide (GIP) receptor deletion leads to reduced bone strength and quality. Bone 56 : 337-342, 2013.

19. Dere E, Huston JP and De Souza Silva MA: The pharmacology, neuroanatomy and neurogenetics of one-trial object recognition in rodents. Neurosci Biobehav Rev 31: 673-704, 2007.

20. Livak KJ and Schmittgen TD: Analysis of relative gene expression data using real-time quantitative PCR and the 2-Delta Delta CT Method. Methods 25: 402-408, 2001.
21. Aggleton JP: One trial object recognition by rats. Q J Exp Psychol 37: 279-294, 1985.

22. Broadbent NJ, Squire LR and Clark RE: Spatial memory, recognition memory and the hippocampus. Learn Mem 17: 5-11, 2010.

23. Heyward FD, Walton RG, Carle MS, et al: Adult mice maintained on a high-fat diet exhibit object location memory deficits and reduced hippocampal SIRT1 gene expression. Neurobiol Learn Mem 98: 25-32, 2012.

24. Hoeffer CA and Klann E: mTOR signaling: at the crossroads of plasticity, memory and disease. Trends Neurosci 33: 67-75, 2010.

25. Michán S, Li Y, Chou MM, et al: SIRT1 is essential for normal cognitive function and synaptic plasticity. J Neurosci 30: 9695-9707, 2010.

26. Gruart A, Sciarretta C, Valenzuela-Harrington M Delgado-García JM and Minichiello L: Mutation at thr TrkB PLC\{gamma\}-docking site affects hippocampal LTP and associative learning in conscious mice. Learn Mem 14: 54-62, 2007.

27. Park HR, Park M, Choi J, Park KY, Chung HY and Lee J: A high-fat diet impairs neurogenesis: involvement of lipid peroxidation and brain-derived neurotrophic factor. Neurosci Lett 4: 235-239, 2010.

28. Acheson A, Conover JC, Fandl JP, et al: A BDNF autocrine loop in adult sensory neurons prevents cell death. Nature 374 : 450-453, 1994.

29. Cao L, Jiao X, Zuzga DS, et al: VEGF links hippocampal activity with neurogenesis, learning and memory. Nat Genet 36: 827-835, 2004.

30. Plaschke K, Staub J, Ernst E and Marti HH: VEGF overexpression improves mice cognitive abilities after unilateral common carotid artery occlusion. Exp Neurol 214: 285-292, 2008. 